\title{
A further step towards a physically consistent virtual crack closure technique
}

\author{
Paolo S. Valvo \\ Phone +39050 2218200 \\ Fax +39050 2218201 \\ E-mail p.valvo@ing.unipi.it
}

University of Pisa, Department of Civil and Industrial Engineering, Largo Lucio Lazzarino, I 56122 Pisa (PI), Italy

\begin{abstract}
The standard virtual crack closure technique may calculate negative values of the modal contributions to the energy release rate when analysing problems with highly asymmetric cracks. To avoid such physically meaningless results, a method is proposed, where the partitioning of fracture modes is based on the decomposition of the crack-tip nodal force into energetically orthogonal components. As an example, a delaminated cantilever beam subjected to bending moments is analysed. Both geometric and algebraic interpretations of the method are discussed.
\end{abstract}

Keywords: mixed-mode fracture, energy release rate, virtual crack closure technique, energetic orthogonality, ellipse of elasticity.

\section{Introduction}

The virtual crack closure technique (VCCT) is a well-established method for calculating the energy release rate, $G$, when analysing fracture problems via the finite element method (Krueger 2004, Krueger et al. 2013). The technique is based on the numerical implementation of the crack closure integral (Irwin 1958), as first proposed for two-dimensional problems by Rybicki and Kanninen (1977) and later extended to three-dimensional problems by Shivakumar et al. (1988).

For mixed-mode fracture problems, such as the delamination of composite materials and interfacial fracture, the energy release rate is the sum of three contributions, $G_{\mathrm{I}}, G_{\mathrm{II}}$, and $G_{\mathrm{III}}$, associated to the three basic fracture modes (I or opening, II or sliding, and III or tearing). According to the standard VCCT, the modal contributions correspond to the amounts of work done to close the extended crack by the Cartesian components of the crack-tip nodal force. In particular, for I/II mixed-mode fracture problems, $G_{\mathrm{I}}$ is related to the work done by the normal crack-tip force component, $Z$, on the corresponding crack-tip opening displacement, $\Delta w$, while $G_{\mathrm{II}}$ is related to the work done by the tangential crack-tip force component, $X$, on the corresponding crack-tip sliding 
displacement, $\Delta u$ (here, normal and tangential refer to the direction of crack propagation). Nevertheless, Valvo (2012) has shown that the standard VCCT may be inappropriate to analyse problems with highly asymmetric cracks, as negative values of $G_{\text {I }}$ and $G_{\text {II }}$ may be calculated. Furthermore, he has found that the origin of such physically meaningless results resides in the lack of energetic orthogonality between the Cartesian components of the crack-tip force used to

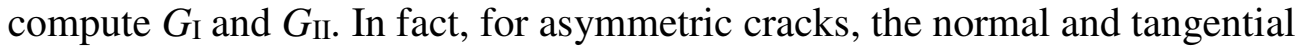
crack-tip force components may cause opening and sliding displacements that give rise to non-zero mutual work (in the sense of Betti's reciprocity theorem). When the mutual work is negative, depending on the geometry and loads of the analysed problem, either $G_{\mathrm{I}}$ or $G_{\mathrm{II}}$ may take on negative values.

To overcome the above described drawback, Valvo (2012) has proposed a revised VCCT, where non-negative $G_{\mathrm{I}}$ and $G_{\mathrm{II}}$ are obtained by associating such quantities to the amounts of work done by two energetically orthogonal -i.e. having a null mutual work - systems of forces. The latter correspond to the components of the crack-tip nodal force along the directions of two conjugate diameters of an ellipse of crack-tip flexibility, similar to the ellipse of elasticity (Culmann 1875). However, since there are infinitely many couples of conjugate diameters - all corresponding to two energetically orthogonal systems of forces there are infinitely many ways to define $G_{\mathrm{I}}$ and $G_{\text {II }}$ as non-negative quantities. The definition adopted by Valvo (2012) leads to the result that pure mode I fracture $\left(G_{\mathrm{II}}=0\right)$ is obtained when the tangential crack-tip force component is zero $(X=0)$, while pure mode II fracture $\left(G_{I}=0\right)$ is obtained when the crack-tip opening displacement is zero $(\Delta w=0)$. Accordingly, however - as the example analysed in this paper will show - there is a range of behaviour where contributions to $G_{\text {I }}$ come also from compressive normal crack-tip force components $(Z<0)$, which should instead be excluded (Fett 2001).

The present brief note aims at making a further step towards the development of a physically consistent virtual crack closure technique. Here, the partitioning of fracture modes proposed by Valvo (2012) is called into question. In its place, a different definition is advanced, based on the assumption that pure mode I fracture corresponds to a null crack-tip sliding displacement $(\Delta u=0)$. Accordingly, $G_{\mathrm{I}}$ and $G_{\mathrm{II}}$ are still associated to the amounts of work done by two energetically orthogonal systems of crack-tip forces. But, pure mode II 
corresponds to a null normal crack-tip force $(Z=0)$. As a result, it is possible to enforce $G_{I}=0$ when $Z<0$ and obtain a 'smooth' transition from the range of I/II mixed-mode behaviour to pure mode II conditions. However, it should be noted that, when $Z<0$, the computation of $G_{\text {II }}$ requires some additional considerations, which are postponed to a forthcoming paper (Valvo 2015).

About the definition of pure fracture modes, it seems appropriate to recall the contributions by Wang and Guan (2012), Wang and Harvey (2012), and Wang et al. (2013). They propose an orthogonal fracture mode partition theory and define two pairs of 'locally' pure modes. Interestingly, their first and second pairs of pure modes respectively correspond to the two, alternative conditions for pure modes obtained in the present work $(\Delta u=0$ and $Z=0)$ and in Valvo (2012) $(X=0$ and $\Delta w=0$ ). It should also be mentioned that Wang and co-workers calculate negative $G_{\mathrm{I}}$ and $G_{\mathrm{II}}$ for load cases falling in between the 'locally' pure modes.

The paper is structured as follows. First, the basics of the virtual crack closure technique are briefly recalled and an expression of $G$ based on the definition of a crack-tip flexibility matrix is deduced. Then, $G_{\mathrm{I}}$ and $G_{\mathrm{II}}$ are defined by associating such quantities to the amounts of work done by the crack-tip forces in an ideal two-step process of closure of the extended crack. As an example, the analysis of a delaminated cantilever beam subjected to bending moments is illustrated. Discussion about possible geometric and algebraic interpretations of the method follows. In particular, the ellipse of crack-tip flexibility is introduced, which helps to visualise the relationship between the directions of the crack-tip force and relative displacement vectors. Furthermore, it is shown how the proposed partitioning of fracture modes corresponds to a particular decomposition of the crack-tip flexibility matrix.

\section{Virtual crack closure technique}

\subsection{Problem formulation}

A two-dimensional (plane stress or plane strain) problem is considered, where a body of width $B$ is affected by a straight crack of length $a$ (Fig. 1a). Suitable static and/or kinematic conditions are prescribed at the body's boundary. The material is assumed to be linearly elastic. A Cartesian reference system, $O x z$, is fixed with the $x$ - and $z$-axes respectively parallel and orthogonal to the crack propagation 
direction. Let $u$ and $w$ denote the displacement components along the $x$ - and $z$ axes, respectively.

The problem is analysed via the finite element method (FEM). In the neighbourhood of the crack tip (Fig. 1b), the body is discretised through a regular mesh of 4-node plane elements of size $\Delta a$ in the $x$-direction. The nodes placed on the fracture surface are orderly labelled with the letters $A, B, C, \ldots$ in the direction of crack advance. Superscripts ${ }^{-}$and ${ }^{+}$respectively denote the nodes on the lower and upper crack faces. Such nodes are initially bonded together by suitable internal constraints, which are progressively released to simulate crack growth. The crack tip is initially located at node $C^{-}$(coincident with $C^{+}$).

(a)

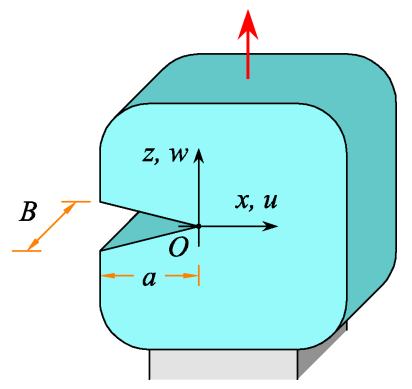

(b)

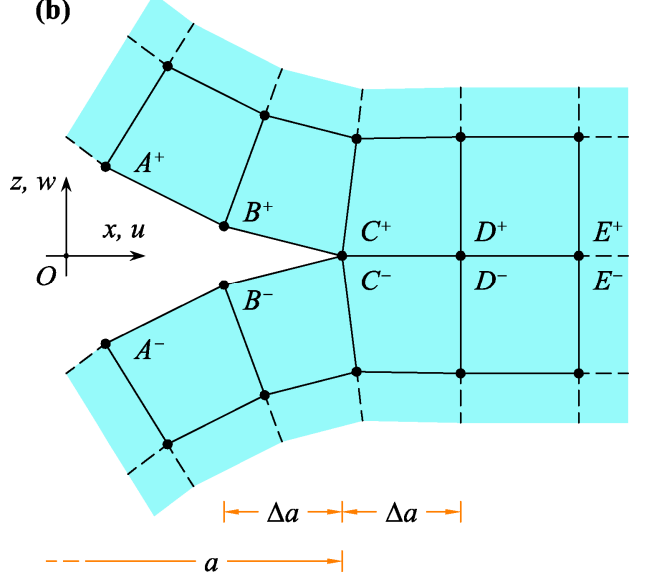

Fig. 1 Problem formulation: a cracked body; b finite element mesh in a neighbourhood of the crack tip

\subsection{Energy release rate}

The energy release rate, $G$, is the total potential energy of the system spent in the crack growth process, per unit area of new surface created. According to Irwin (1958), the energy spent to produce an extension of the crack is equal to the work done to close the crack by the forces acting on the crack faces prior to crack extension. Within the adopted FEM framework, Irwin's concept yields

$$
G=\frac{1}{2 B \Delta a}(X \Delta u+Z \Delta w)
$$

where $X$ and $Z$ respectively are the tangential ( $x$-axis) and normal ( $z$-axis) components of the crack-tip nodal force (Fig. 2a), while $\Delta u$ and $\Delta w$ are the corresponding relative displacements (Fig. 2b) (Rybicki and Kanninen 1977, Krueger 2004). 
(a)

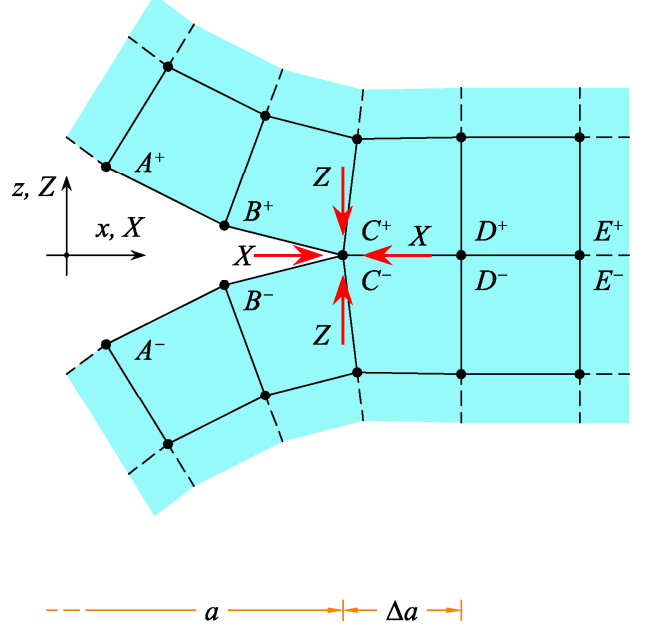

(b)

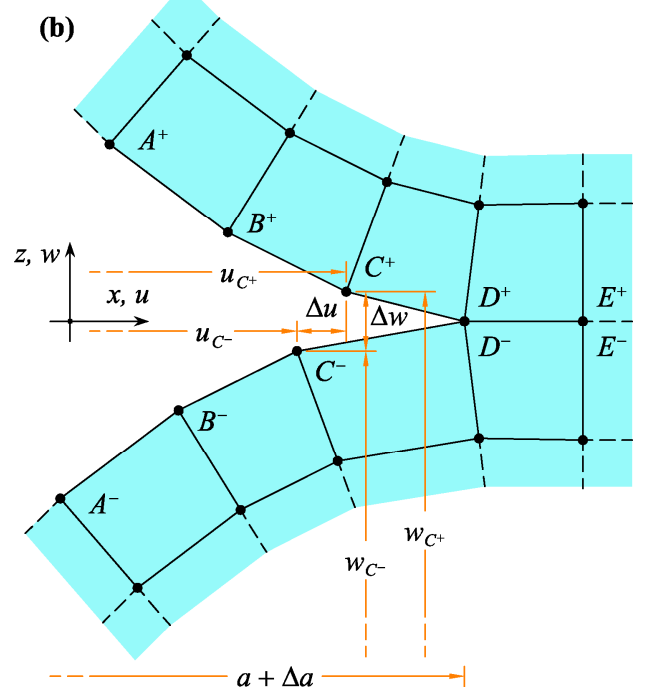

Fig. 2 Virtual crack closure technique: a crack-tip forces; b crack-tip relative displacements

The relative displacements caused by crack advance are equal in magnitude (and opposite in sign) to the relative displacements produced by application of the crack-tip forces. Thus, for a linearly elastic body it turns out that $\Delta u=f_{x x} X+f_{x z} Z$ and $\Delta w=f_{z x} X+f_{z z} Z$

where $f_{x x}, f_{x z}, f_{z x}$, and $f_{z z}$ are flexibility coefficients, equal to the crack-tip relative displacements produced by unit forces applied at nodes $C^{+}$and $C^{-}$(Fig. 3). The flexibility coefficients can be computed by conducting two preliminary analyses on the finite element mesh with the extended crack (Valvo 2012). It is noted that the coefficient $f_{x z}$ ( $=f_{z x}$ by virtue of Betti's reciprocity theorem) expresses an elastic coupling between the crack-tip force in the $x$-direction and the relative displacement in the $z$-direction and, vice versa, between the crack-tip force in the $z$-direction and the relative displacement in the $x$-direction. This coupling vanishes $\left(f_{x z}=0\right)$ for bodies with symmetric cracks, but is generally present $\left(f_{x z} \neq 0\right)$ for bodies with asymmetric cracks. 

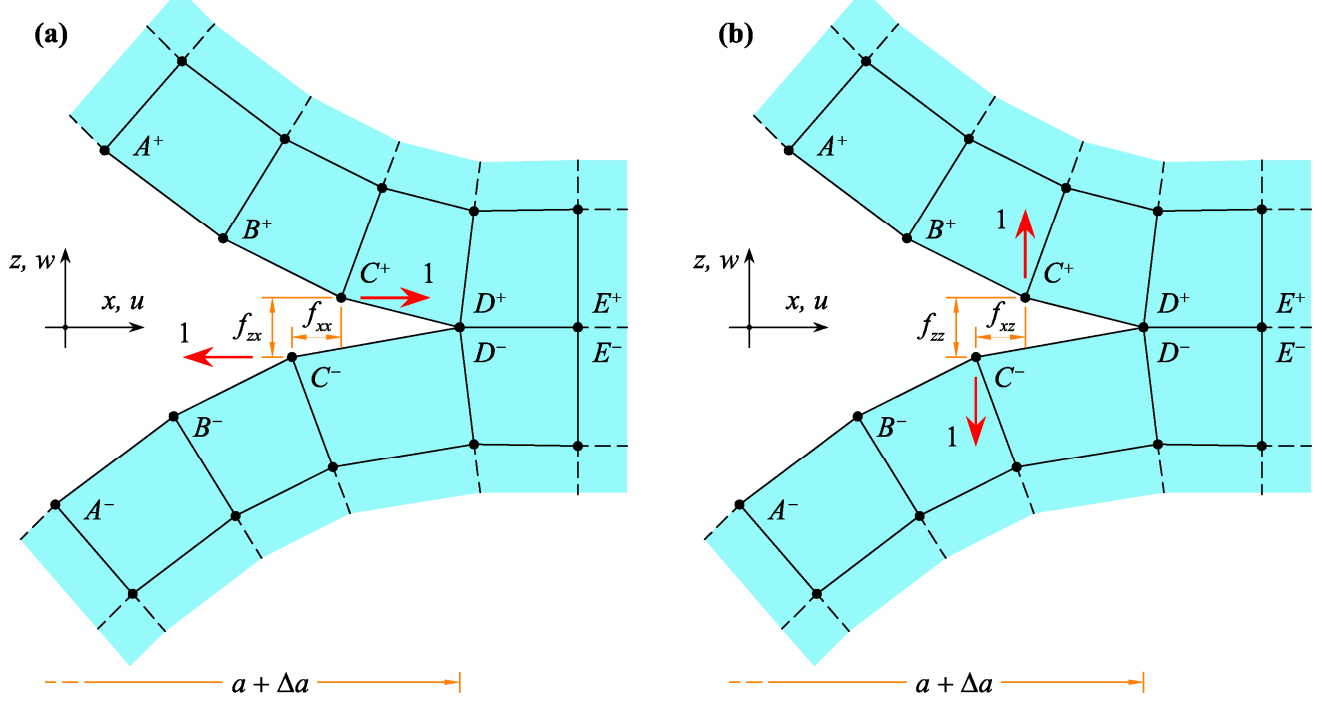

Fig. 3 Flexibility coefficients: a unit forces in the $x$-direction; b unit forces in the $z$-direction

It is convenient to introduce vector notation by collecting the crack-tip force and displacement components into the crack-tip force vector, $\mathbf{r}=(X, Z)^{\mathrm{T}}$, and crack-tip relative displacement vector, $\Delta \mathbf{s}=(\Delta u, \Delta w)^{\mathrm{T}}$, respectively (superscript ${ }^{\mathrm{T}}$ denotes the matrix transpose operation). As a consequence, Eqs. (2) can be written compactly as

$\Delta \mathbf{s}=\mathbf{F r}$,

where

$\mathbf{F}=\left[\begin{array}{ll}f_{x x} & f_{x z} \\ f_{z x} & f_{z z}\end{array}\right]$

is the (symmetric) crack-tip flexibility matrix. With Eqs. (3) and (4), the expression for the energy release rate Eq. (1) becomes

$G=\frac{1}{2 B \Delta a} \mathbf{r}^{\mathrm{T}} \Delta \mathbf{s}=\frac{1}{2 B \Delta a} \mathbf{r}^{\mathrm{T}} \mathbf{F} \mathbf{r}=\frac{1}{2 B \Delta a}(X, Z)\left[\begin{array}{ll}f_{x x} & f_{x z} \\ f_{z x} & f_{z z}\end{array}\right]\left\{\begin{array}{l}X \\ Z\end{array}\right\}$.

It is worth noting that, because of its physical meaning, $G$ is a nonnegative quantity. Hence, $\mathbf{F}$ is a positive definite matrix, which implies $f_{x x}>0$ and $\operatorname{det}(\mathbf{F})=f_{x x} f_{z z}-f_{x z}^{2}>0$.

\subsection{Fracture mode partitioning}

According to Rybicki and Kanninen (1977), the modal contributions to $G$ correspond to the two addends in parenthesis in Eq. (1): 
$G_{\mathrm{I}}=\frac{Z \Delta w}{2 B \Delta a} \quad$ and $\quad G_{\mathrm{II}}=\frac{X \Delta u}{2 B \Delta a}$

However, a physically meaningful partitioning of fracture modes requires decomposing the energy release rate into the sum of two non-negative modal contributions. Instead, Valvo (2012) has demonstrated that Eqs. (7) may yield

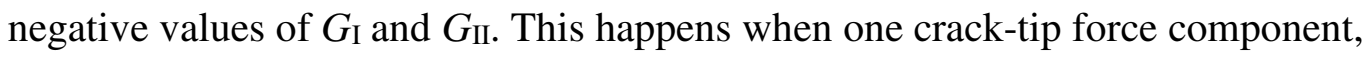
$X$ or $Z$, is opposite in sign with respect to the corresponding relative displacement, $\Delta u$ or $\Delta w$, and thus does negative work in closing the crack (Wang and Guan 2012). In particular, this shortcoming has been revealed in the analysis of bodies with highly asymmetric cracks. In this respect, it should be noted that Rybicki and Kanninen (1977) - following Irwin (1958) - have obtained Eqs. (7) having in mind Westergaard's (1939) solution for the problem of a single straight crack in an infinite plane body made of a linearly elastic, isotropic, and homogeneous material. In this case, the system of acting forces can be decomposed into the sum of a symmetric part and an antisymmetric part (with respect to the crack plane), respectively related to fracture modes I and II. Such a decomposition leads to the correct partitioning of fracture modes for symmetrically cracked bodies. However, it makes little sense in the case of bodies with asymmetric cracks (including bimaterial interface cracks), for which it is not surprising that Eqs. (7) may be no longer valid.

As an alternative, fracture mode partitioning is based here on the assumption that pure mode I fracture corresponds to a null crack-tip sliding displacement $(\Delta u=0)$. As a consequence, the mode II contribution to $G$ will be related to the closure of $\Delta u$, while the mode I contribution will be given by the difference between the total energy release rate and the mode II contribution. In practice, $G_{\mathrm{I}}$ and $G_{\mathrm{II}}$ can be calculated as associated to the amounts of work done by the crack-tip force components in an ideal two-step process of closure of the extended crack. Starting from the fully open crack (Fig. 4a), in the first ideal step, corresponding to the mode II contribution, the crack-tip sliding displacement, $\Delta u$, is closed by applying a suitable tangential crack-tip force, $X_{\text {II }}$, and a null normal crack-tip force (Fig. 4b). Eqs. (2) show that the necessary forces are

$$
X_{\mathrm{II}}=\frac{\Delta u}{f_{x x}}=X+\frac{f_{x z}}{f_{x x}} Z \text { and } \quad Z_{\mathrm{II}}=0
$$


which produce the relative displacements

$\Delta u_{\mathrm{II}}=\Delta u \quad$ and $\quad \Delta w_{\mathrm{II}}=\frac{f_{x z}}{f_{x x}} \Delta u$

In the second ideal step, corresponding to the mode I contribution, the remainders of the crack-tip forces (Fig. 4c),

$X_{\mathrm{I}}=X-X_{\mathrm{II}}=-\frac{f_{x z}}{f_{x x}} Z$ and $\quad Z_{\mathrm{I}}=Z-Z_{\mathrm{II}}=Z$,

are applied to the crack-tip nodes, which - according to Eqs. (2) - undergo the relative displacements

$\Delta u_{\mathrm{I}}=0 \quad$ and $\quad \Delta w_{\mathrm{I}}=\Delta w-\Delta w_{\mathrm{II}}=\frac{1}{f_{x x}}\left(f_{x x} f_{z z}-f_{x z}^{2}\right) Z$
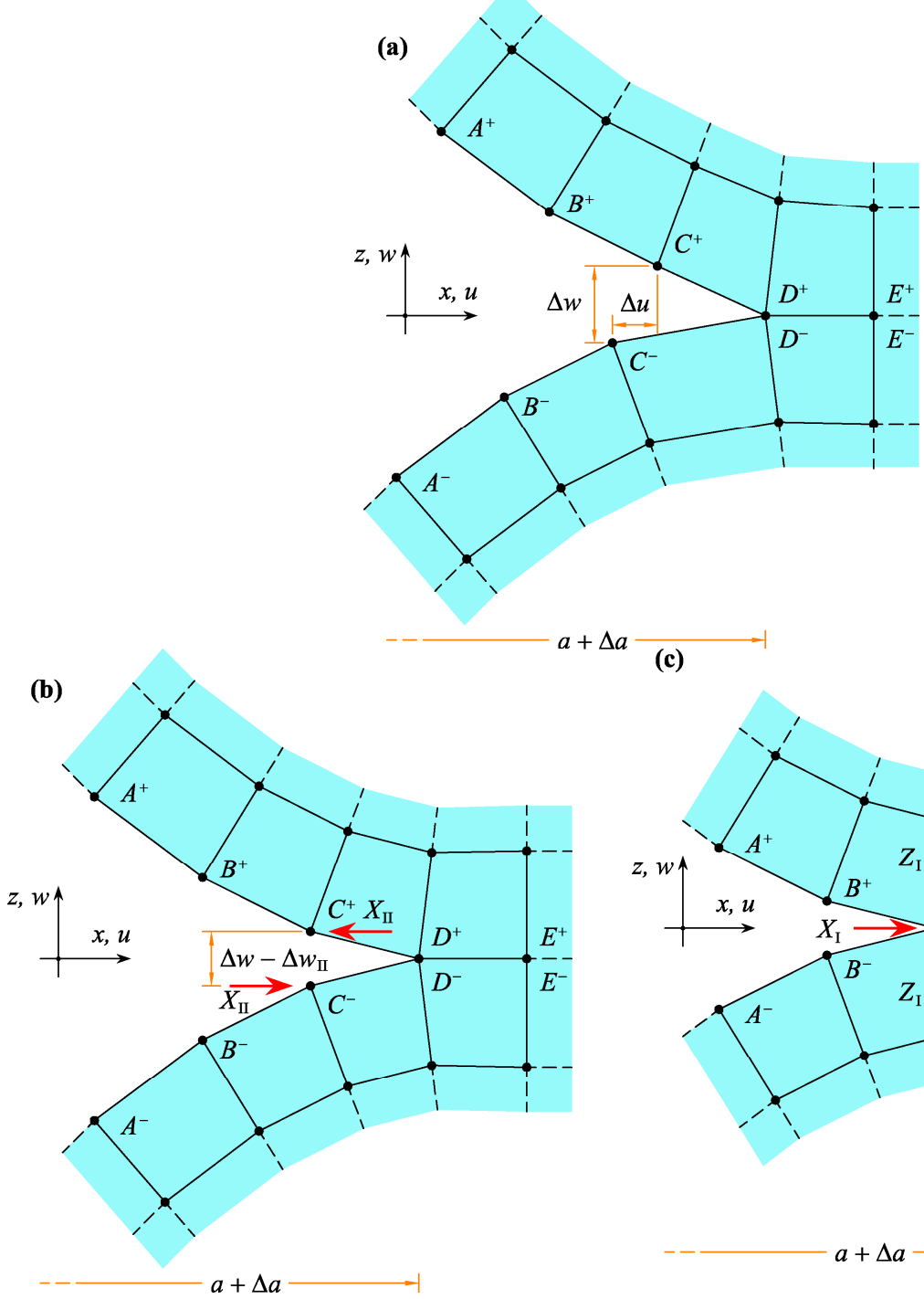

(c)

Fig. 4 Fracture mode partitioning: a fully open crack; b step 1) mode II contribution, residual crack-tip opening displacement; c step 2) mode I contribution, complete crack closure 
Eqs. (9) show that in the first ideal step the gap in the $x$-direction, $\Delta u$, is completely closed, while the gap in the $z$-direction, $\Delta w$, may be partly closed (if $f_{x z} \Delta u>0$ ) or further opened (if $f_{x z} \Delta u<0$ ). Eqs. (11) indicate that in the second ideal step the gap in the $x$-direction is not altered, while the residual gap in the $z$ direction, $\Delta w-\Delta w_{\text {II }}$, is closed.

According to the above, the mode I and II contributions to the energy release rate respectively correspond to the amounts of work done by the force component $Z_{\mathrm{I}}$ on the displacement $\Delta w_{\mathrm{I}}$ and by $X_{\mathrm{II}}$ on $\Delta u_{\mathrm{II}}$ :

$G_{\mathrm{I}}=\frac{Z_{\mathrm{I}} \Delta w_{\mathrm{I}}}{2 B \Delta a} \quad$ and $\quad G_{\mathrm{II}}=\frac{X_{\mathrm{II}} \Delta u_{\mathrm{II}}}{2 B \Delta a}$

By substituting Eqs. (8)-(11) into (12), the following expressions for the modal contributions are obtained:

$G_{\mathrm{I}}=\frac{1}{2 B \Delta a} \frac{1}{f_{x x}}\left(f_{x x} f_{z z}-f_{x z}^{2}\right) Z^{2} \quad$ and $\quad G_{\mathrm{II}}=\frac{1}{2 B \Delta a} \frac{1}{f_{x x}}\left(f_{x x} X+f_{x z} Z\right)^{2}$.

Eqs. (13) - by recalling also Eqs. (6) - show that the present assumption on fracture mode partitioning leads to calculate both $G_{\mathrm{I}}$ and $G_{\mathrm{II}}$ as non-negative quantities. This result can be regarded as a consequence of the energetic orthogonality of the systems of forces defined by Eqs. (8) and (10). Actually, it is an easy task to demonstrate that they give rise to a null mutual work, $X_{\mathrm{I}} \Delta u_{\mathrm{II}}+Z_{\mathrm{I}} \Delta w_{\mathrm{II}}=X_{\mathrm{II}} \Delta u_{\mathrm{I}}+Z_{\mathrm{II}} \Delta w_{\mathrm{I}}=0$.

For implementation, it is also convenient to express $G_{\text {I }}$ and $G_{\text {II }}$ as functions of the displacements only. To this aim, by inverting Eqs. (2) and substituting the result into (13), the following expressions are obtained:

$G_{\mathrm{I}}=\frac{1}{2 B \Delta a} \frac{1}{f_{x x}} \frac{\left(f_{x z} \Delta u-f_{x x} \Delta w\right)^{2}}{f_{x x} f_{z z}-f_{x z}^{2}}$ and $G_{\mathrm{II}}=\frac{1}{2 B \Delta a} \frac{\Delta u^{2}}{f_{x x}}$

Eqs. (13) and (14) also reveal the conditions for pure fracture modes: pure mode I $\left(G_{\mathrm{II}}=0\right)$ is obtained when $\Delta u=0$, pure mode II $\left(G_{\mathrm{I}}=0\right)$ when $Z=0$.

Lastly, it is noted that for $f_{x z}=0$, Eqs. (8) and (11) yield $X_{\mathrm{II}}=X$ and $\Delta w_{\mathrm{I}}=\Delta w$. In this case - since from Eqs. (9) and (10) also $\Delta u_{\mathrm{II}}=\Delta u$ and $Z_{\mathrm{I}}=Z-$ Eqs. (7) and (12) coincide, hence the proposed method reduces to the standard VCCT. This happens, in particular, for bodies with symmetric cracks. 


\section{Example}

\subsection{Finite element analysis}

As an illustrative example, the method is applied to the problem of a delaminated cantilever beam subjected to bending moments, $M_{1}$ and $M_{2}$, on its upper and lower arms, respectively (Fig. 5a). The beam has length $L=100 \mathrm{~mm}$, width $B=25 \mathrm{~mm}$, and thickness $H=10 \mathrm{~mm}$. The delamination length is $a=50 \mathrm{~mm}$. The two arms have thicknesses $H_{1}=0.5 \mathrm{~mm}$ and $H_{2}=9.5 \mathrm{~mm}$. The material is linearly elastic, isotropic, and homogeneous, with Young's modulus $E=100 \mathrm{GPa}$ and Poisson's ratio $v=0.3$. A finite element analysis of the problem has been carried out using the commercial software Abaqus 6.9. The beam has been modelled with 4-node linear plane stress (CPS4) elements (Fig. 5b). The element size in the crack-tip region is $\Delta a=0.10 \mathrm{~mm}$ (Fig. 5c). The present analysis does not account for contact and interpenetration constraints, which means that the crack faces may overlap freely. Such constraints will be instead considered in a forthcoming paper (Valvo 2015).

(a)

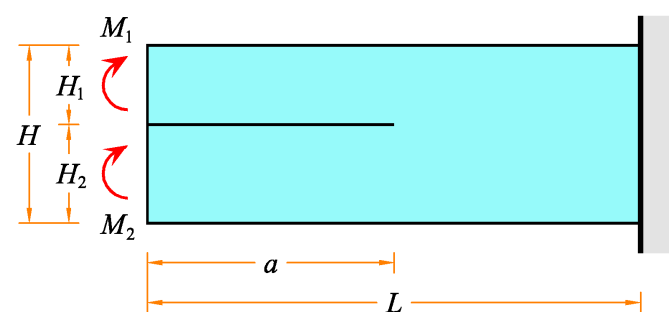

(b)

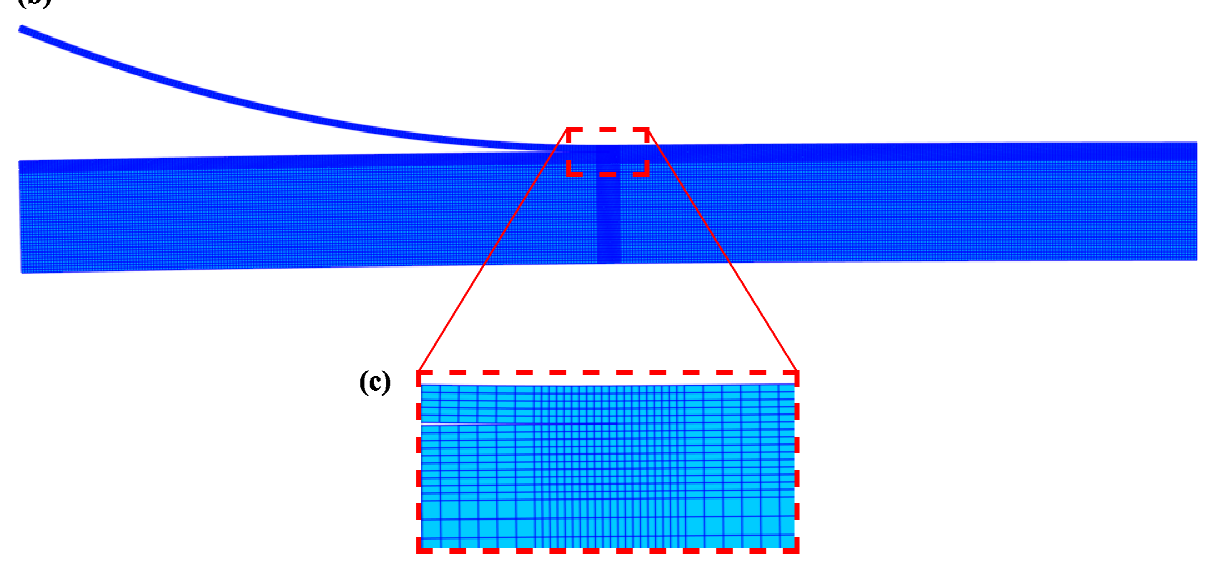

Fig. 5 Delaminated cantilever beam subjected to bending moments: a geometry and loads; b FEM model; c detail of the mesh at the crack tip 


\subsection{Results}

In order to explore a wide range of mode mixities, $M_{1}=1 \mathrm{~N} \mathrm{~m}$ is kept fixed, while $M_{2}$ varies. Figures $6 \mathrm{a}$ and $6 \mathrm{~b}$ respectively show the normal and tangential components of the crack-tip nodal force, $X$ and $Z$, and crack-tip relative displacements, $\Delta u$ and $\Delta w$, as functions of $M_{2}$. Figure $6 \mathrm{c}$ shows the mode I and II contributions to the energy release rate, $G_{\text {I }}$ and $G_{\text {II }}$, as functions of $M_{2}$. Continuous lines correspond to the present method, Eqs. (14). Dotted lines correspond to the standard VCCT, Eqs. (7).

The standard VCCT predicts pure mode I conditions $\left(G_{\mathrm{II}}=0\right)$ for two distinct values of $M_{2}$, corresponding to $\Delta u=0$ and $X=0$, and negative mode II contribution $\left(G_{\mathrm{II}}<0\right)$ for $M_{2}$ in the range between those values. Likewise, it predicts pure mode II conditions $\left(G_{\mathrm{I}}=0\right)$ for $M_{2}$ corresponding to $Z=0$ and $\Delta w=0$ and negative mode I contribution $\left(G_{\mathrm{I}}<0\right)$ for $M_{2}$ in the range between. Instead, the present method furnishes always non negative values of $G_{\mathrm{I}}$ and $G_{\mathrm{II}}$ and predicts pure mode I and II conditions for $M_{2}=M_{2 \mathrm{I}} \cong-187.67 \mathrm{~N} \mathrm{~m}(\Delta u=0)$ and $M_{2}=M_{2 \mathrm{II}} \cong 249.51 \mathrm{~N} \mathrm{~m}(Z=0)$, respectively.

Despite contact and interpenetration of the crack faces have not been modelled in the present FEM analysis, some comments can be made. As can be noted from figure $6 \mathrm{a}$, for $M_{2}>M_{2 \mathrm{II}}$, the normal crack-tip force component is compressive $(Z<0)$. However, figure $6 \mathrm{~b}$ also shows that right of $M_{2 I I}$ there is a (small) range of values of $M_{2}$, for which the crack faces open $(\Delta w>0)$, before entering the interpenetration region $(\Delta w<0)$. In this range, according to Valvo (2012), fracture would occur in I/II mixed-mode, with a $G_{I}$ contribution stemming from a negative normal crack-tip force. Instead, according to the present method, pure mode II can be enforced by setting $G_{\mathrm{I}}=0$ for $Z<0$ (Fig. 6c). The transition from the range of I/II mixed-mode behaviour to pure mode II conditions turn out to be 'smooth' (i.e. there is continuous join between the plots of $G_{\mathrm{I}}$ left and right of $\left.M_{2 I I}\right)$. It should be noted, however, that the expressions derived in Section 2.3 are not generally valid in this range of behaviour, so that a different derivation for $G_{\text {II }}$ is needed for $M_{2}>M_{2 I I}($ Valvo 2015). 


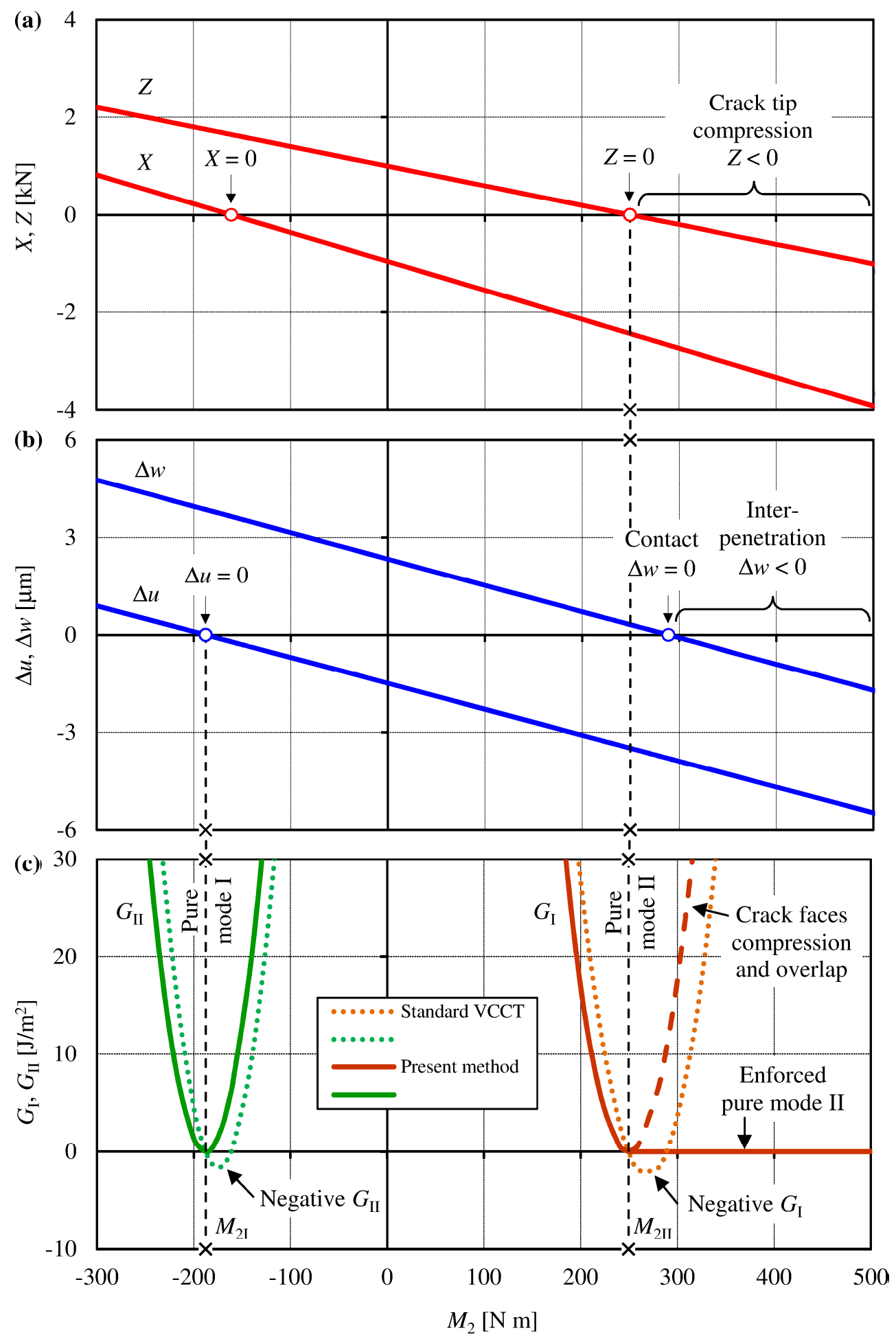

Fig. 6 Results for the delaminated cantilever beam: a crack-tip nodal force components; $\mathbf{b}$ cracktip relative displacements; c energy release rate contributions 


\section{Discussion}

\subsection{Ellipse of crack-tip flexibility}

A geometric construction can help to shed light on the matter at hand. To this aim, the conic section associated to the crack-tip flexibility matrix, $\mathbf{F}$, is considered. It is defined by the equation

$f_{x x} x^{2}+2 f_{x z} x z+f_{z z} z^{2}-1=0$

in the $O x z$-plane. Since $\operatorname{det}(\mathbf{F})>0$ (recall Eqs. (6)), this conic section turns out to be an ellipse, $\Gamma$, termed the ellipse of crack-tip flexibility (Valvo 2012). The ellipse's centre coincides with the origin of the reference system, $O x z$. Its major and minor axes, $a$ and $b$, are rotated by an angle $\phi$ (different from 0 unless $f_{x z}=0$ ) with respect to the reference axes (Fig. 7a).

The ellipse of crack-tip flexibility shares many properties with Culmann's (1875) ellipse of elasticity. The latter can be used to visualise the direction of the displacement caused by the application of a force to an elastic body. Likewise, the ellipse of crack-tip flexibility enables visualisation of the relationship between the directions of the crack-tip force vector, $\mathbf{r}$, and relative displacement vector, $\Delta \mathbf{s}$. In fact, let $r$ and $s$ be the ellipse's diameters respectively parallel to $\mathbf{r}$ and $\Delta \mathbf{s}$ (Fig. $7 b$ ). In addition, let $t$ be the diameter conjugate to $r$ (i.e. the diameter which is parallel to the tangents, $t^{\prime}$ and $t^{\prime \prime}$, to the ellipse at the endpoints, $P^{\prime}$ and $P^{\prime \prime}$, of the diameter $r$ ). Valvo (2012) has demonstrated that $s$ is orthogonal to $t$. This means the direction of the crack-tip displacement vector, $\Delta \mathbf{s}$, can be obtained as follows. Given the crack-tip force vector, $\mathbf{r}$, trace the diameter $r$. Let $\mathbf{n}$ be the outer normal to the ellipse at $P^{\prime}$, the endpoint of $r$ in the direction of $\mathbf{r}$. The direction of $\Delta \mathbf{s}$ will be the same as the direction of $\mathbf{n}$.

The above described graphic construction can be applied to better understand the meaning of the two-step crack closure process defined in Section 2.3. To this aim, the crack-tip force components defined by Eqs. (8) and (10) are collected into two crack-tip force vectors,

$\mathbf{r}_{\mathrm{I}}=\left\{\begin{array}{l}X_{\mathrm{I}} \\ Z_{\mathrm{I}}\end{array}\right\}=\left\{\begin{array}{c}-\frac{f_{x z}}{f_{x x}} \\ Z\end{array}\right\} \quad$ and $\quad \mathbf{r}_{\mathrm{II}}=\left\{\begin{array}{l}X_{\mathrm{II}} \\ Z_{\mathrm{II}}\end{array}\right\}=\left\{\begin{array}{c}X+\frac{f_{x z}}{f_{x x}} Z \\ 0\end{array}\right\}$. 
The vectors $\mathbf{r}_{\text {II }}$ and $\mathbf{r}_{\text {I }}$ respectively are the components of $\mathbf{r}$ along the directions of the $x$-axis and a new axis denoted with $\bar{z}$. Such axes intersect the ellipse at points $P_{\mathrm{II}}$ and $P_{\mathrm{I}}$, respectively (Fig. 7c). In particular, the $\bar{z}$-axis is such that the outer normal to the ellipse at $P_{\mathrm{I}}$ is orthogonal to the $x$-axis (which implies that the $x$ - and $\bar{z}$-axes have the directions of two conjugate diameters of the ellipse). During the first of the two crack closure steps, the application of the force $\mathbf{r}_{\text {II }}$ at the crack-tip nodes produces the displacement $\Delta \mathbf{s}_{\text {II }}$ (parallel to the outer normal to the ellipse at $\left.P_{\mathrm{II}}\right)$. During the second step, the application of $\mathbf{r}_{\mathrm{I}}$ produces the displacement $\Delta \mathbf{s}_{\mathrm{I}}$ (parallel to the outer normal to the ellipse at $P_{\mathrm{I}}$ ). It can be verified that $\Delta \mathbf{s}_{\mathrm{I}}$ is orthogonal to $\mathbf{r}_{\mathrm{II}}$, while $\Delta \mathbf{s}_{\mathrm{II}}$ is orthogonal to $\mathbf{r}_{\mathrm{I}}$. Consequently, the mutual work done by $\mathbf{r}_{\text {I }}$ on $\Delta \mathbf{s}_{\text {II }}$ is zero, as well as the work done by $\mathbf{r}_{\text {II }}$ on $\Delta \mathbf{s}_{\text {I }}$. In other words, $\mathbf{r}_{I}$ and $\mathbf{r}_{I I}$ are energetically orthogonal, as anticipated in Section 2.3. This result is a consequence of the fact that the $x$-and $\bar{z}$-axes have the directions of two conjugate diameters of the ellipse. Actually, energetic orthogonality would be obtained as well, if $\mathbf{r}$ was decomposed along the directions of any other two conjugate diameters.

(a)

(b)

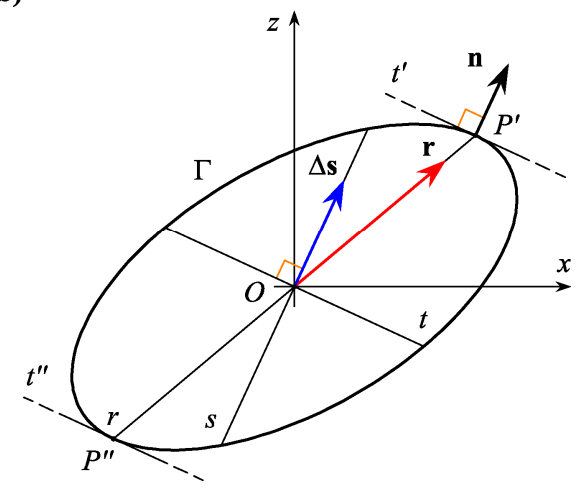

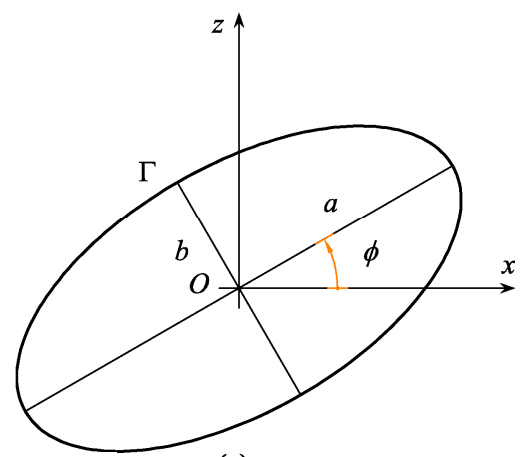

(c)

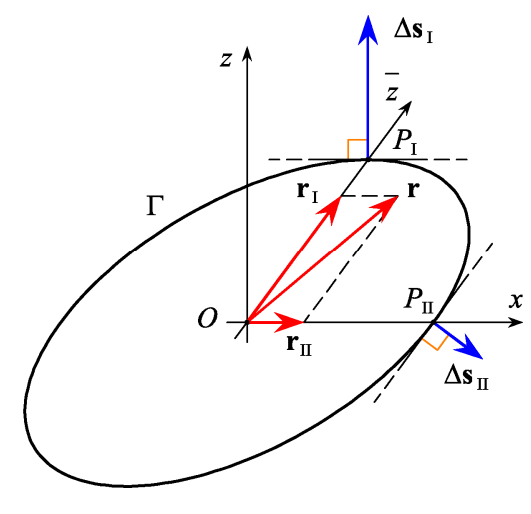

Fig. 7 Ellipse of crack-tip flexibility: a definition; b crack-tip force and relative displacement vectors; $\mathbf{c}$ decomposition of the crack-tip force vector into energetically orthogonal components

The ellipse of crack-tip flexibility can also be used to determine graphically the acting fracture mode in a given problem (Fig. 8). When the 
direction of the crack-tip force vector, $\mathbf{r}$, coincides with the $\bar{z}$-axis, fracture occurs in mode I. When $\mathbf{r}$ lies on the $x$-axis, fracture occurs in mode II. All the other directions of $\mathbf{r}$ correspond to I/II mixed-mode fracture. Furthermore, contact and interpenetration can be detected. To this aim, the $\bar{x}$-axis is introduced, which intersects the ellipse at the points where the outer normal, $\mathbf{n}$, is orthogonal to the $z$-axis (it turns out that the $\bar{x}$ - and $z$-axes have the directions of two conjugate diameters of the ellipse). When $\mathbf{r}$ has the direction of the $\bar{x}$-axis, contact between the crack-tip nodes occurs $(\Delta w=0)$. When $\mathbf{r}$ falls in the region below the $\bar{x}$-axis, interpenetration of the crack faces is expected $(\Delta w<0)$. Lastly, it is noted that when $\mathbf{r}$ falls in the region below the $x$-axis, the normal crack-tip force is compressive $(Z<0)$. In this case, as explained at the end of Section 3.2, pure mode II can be enforced by setting $G_{\mathrm{I}}=0$, but the computation of $G_{\mathrm{II}}$ requires a more refined analysis (Valvo 2015).

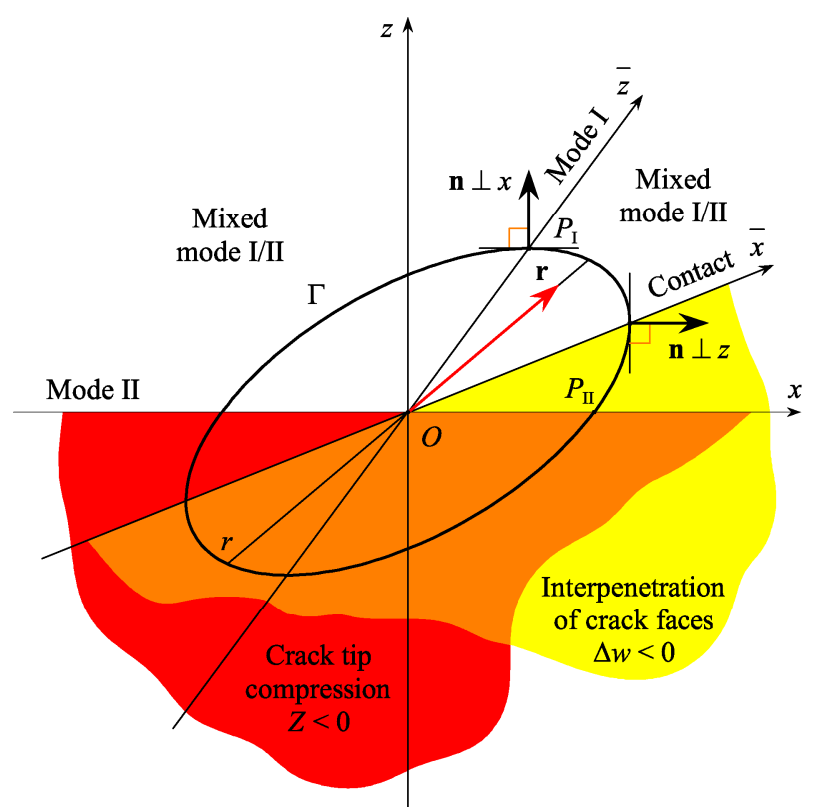

Fig. 8 Ellipse of crack-tip flexibility: determination of fracture mode

\subsection{Decomposition of the flexibility matrix}

Besides the geometric interpretation illustrated in Section 4.1, it will be useful to discuss also a possible algebraic interpretation of the proposed method. To this aim, it is observed that the partitioning of fracture modes described in Section 2.3 corresponds to the Cholesky decomposition of the crack-tip flexibility matrix: $\mathbf{F}=\mathbf{U}^{\mathrm{T}} \mathbf{D} \mathbf{U}$,

where 


$$
\mathbf{U}=\left[\begin{array}{cc}
1 & \frac{f_{x z}}{f_{x x}} \\
0 & 1
\end{array}\right]
$$

is a dimensionless, unit upper triangular matrix and

$$
\mathbf{D}=\left[\begin{array}{cc}
f_{x x} & 0 \\
0 & f_{z z}-\frac{f_{x z}^{2}}{f_{x x}}
\end{array}\right]=\left[\begin{array}{cc}
f_{\mathrm{II}} & 0 \\
0 & f_{\mathrm{I}}
\end{array}\right]
$$

is a diagonal flexibility matrix. By substituting Eq. (17) into (5), the expression for the energy release rate becomes

$$
G=\frac{1}{2 B \Delta a}(\mathbf{U r})^{\mathrm{T}} \mathbf{D} \mathbf{U} \mathbf{r}=\frac{1}{2 B \Delta a}\left(\mathbf{r}^{*}\right)^{\mathrm{T}} \mathbf{D} \mathbf{r}^{*},
$$

where

$$
\mathbf{r}^{*}=\left\{\begin{array}{l}
r_{\mathrm{II}} \\
r_{\mathrm{I}}
\end{array}\right\}=\mathbf{U} \mathbf{r}=\left[\begin{array}{cc}
1 & \frac{f_{x z}}{f_{x x}} \\
0 & 1
\end{array}\right]\left\{\begin{array}{l}
X \\
Z
\end{array}\right\}=\left\{\begin{array}{c}
X+\frac{f_{x z}}{f_{x x}} \\
Z
\end{array}\right\}
$$

is a 'corrected' crack-tip force vector.

With Eqs. (19) and (21), the energy release rate Eq. (20) becomes

$$
G=\frac{1}{2 B \Delta a} f_{\mathrm{I}} r_{\mathrm{I}}^{2}+\frac{1}{2 B \Delta a} f_{\mathrm{II}} r_{\mathrm{II}}^{2} .
$$

It can be easily verified that the two addends in Eq. (22) correspond to the modal contributions, $G_{\mathrm{I}}$ and $G_{\mathrm{II}}$, as given by Eqs. (13).

The illustrated algebraic interpretation is the basis to extend the method to three-dimensional problems involving I/II/III mixed-mode fracture (Valvo 2014).

\section{$5 \quad$ Concluding remarks}

A modified virtual crack closure technique has been presented for calculating the energy release rate, $G$, and its modal contributions, $G_{\mathrm{I}}$ and $G_{\mathrm{II}}$, in I/II mixed-mode fracture problems. The proposed method overcomes a shortcoming of the standard VCCT, which may calculate physically meaningless, negative values of $G_{\text {I }}$ and $G_{\text {II }}$ when analysing problems with highly asymmetric cracks. The method is based on the decomposition of the crack-tip nodal force into energetically orthogonal components and the assumption that pure mode I fracture corresponds to a null 
crack-tip sliding displacement $(\Delta u=0)$. As a result, the condition for pure mode II fracture is a null normal crack-tip force $(Z=0)$. Thus, it is possible to enforce mode II conditions $\left(G_{\mathrm{I}}=0\right)$ when the normal crack-tip force is compressive $(Z<0)$ and obtain a 'smooth' transition from the range of I/II mixed-mode behaviour to pure mode II conditions. As an example, the analysis of a delaminated cantilever beam has been presented. Lastly, both geometric and algebraic interpretations of the method have been discussed.

The presented method and results can be considered as a continuation of the work started by Valvo (2012) towards the development of a physically consistent VCCT. But, further work is necessary, for instance, to include the effects of contact and friction between the crack faces in the analysis (Laursen 2002), as well as to extend the method to bimaterial interface cracks (Agrawal and Karlsson 2006, Krueger et al. 2013) and three-dimensional problems (Valvo 2014). A full paper accounting for some of the aforementioned topics is currently in preparation (Valvo 2015).

Acknowledgements The revised version of this manuscript was prepared during the author's one semester sabbatical (September 2014-February 2015) at the Technical University of Denmark, DTU Wind Energy, Composite Materials Section. The author wishes to thank all the Section's staff, in particular the Head of Section Professor Bent F. Sørensen, for hospitality, support, and scientific advice during this period.

\section{References}

Agrawal A, Karlsson AM (2006) Obtaining mode mixity for a bimaterial interface crack using the virtual crack closure technique. Int J Fract 141(1-2):75-98. doi: 10.1007/s10704-006-0069$\underline{4}$

Culmann K (1875) Die graphische Statik, 2nd edn. Meyer \& Zeller, Zurich

Irwin GR (1958) Fracture. In: Flügge S (ed) Handbuch der Physik, vol. VI. Springer, Berlin, pp. $551-590$

Fett T (2001) Mixed-mode stress intensity factors for partially opened cracks. Int J Fract 111(4): L67-L72. doi: 10.1023/A:1012473700907

Krueger R (2004) Virtual crack closure technique: History, approach, and applications. Appl Mech Rev 57(2):109-143. doi: $10.1115 / 1.1595677$

Krueger R, Shivakumar K, Raju IS (2013) Fracture mechanics analyses for interface crack problems. A review. In: 54th AIAA/ASME/ASCE/AHS/ASC Structures, Structural Dynamics, and Materials Conference, April 8-11, 2013, Boston, MA. doi: 10.2514/6.20131476 
Laursen TA (2002) Computational contact and impact mechanics: fundamentals of modeling interfacial phenomena in nonlinear finite element analysis. Springer, Berlin

Rybicki EF, Kanninen MF (1977) A finite element calculation of stress intensity factors by a modified crack closure integral. Eng Fract Mech 9(4):931-938. doi: 10.1016/00137944(77)90013-3

Shivakumar KN, Tan PW, Newman JC (1988) A virtual crack-closure technique for calculating stress intensity factors for cracked three dimensional bodies. Int J Fract 36(3):R43-R50. doi: $\underline{10.1007 / \mathrm{BF} 00035103}$

Valvo PS (2012) A revised virtual crack closure technique for physically consistent fracture mode partitioning. Int J Fract 173(1):1-20. doi: 10.1007/s10704-011-9658-y

Valvo PS (2014) A physically consistent virtual crack closure technique for I/II/III mixed-mode fracture problems. Proc Mater Sci 3:1983-1987. doi: 10.1016/j.mspro.2014.06.319

Valvo PS (2015) A physically consistent virtual crack closure technique accounting for contact and interpenetration. Int $\mathrm{J}$ Fract (in preparation)

Wang S, Guan L (2012) On fracture mode partition theories. Comput Mater Sci 52(1):240-245. doi: $10.1016 /$ j.commatsci.2011.03.021

Wang S, Harvey CM (2012) Mixed mode partition theories for one dimensional fracture. Eng Fract Mech 79:329-352. doi: 10.1016/j.engfracmech.2011.11.013

Wang S, Harvey CM, Guan L (2013) Partition of mixed modes in layered isotropic double cantilever beams with non-rigid cohesive interfaces. Eng Fract Mech 111:1-25. doi: $\underline{10.1016 / j . e n g f r a c m e c h .2013 .09 .005 ~}$

Westergaard HM (1939). Bearing pressures and cracks. J Appl Mech Trans ASME 6:A49-A53 